\title{
Dual Polygonal Billiards and Necklace Dynamics
}

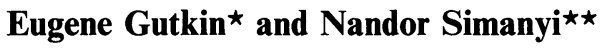 \\ Department of Mathematics, University of Southern California, Los Angeles, CA 90089, USA
}

Received June 15, 1990

\begin{abstract}
We study the orbits of the dual billiard map about a polygonal table using the technique of necklace dynamics. Our main result is that for a certain class of tables, called the quasi-rational polygons, the dual billiard orbits are bounded. This implies that for the subset of rational tables (i.e. polygons with rational vertices) the dual billiard orbits are periodic.
\end{abstract}

\section{Introduction}

Let $P$ be a closed bounded domain in $\mathbf{R}^{2}$ with a $C^{1}$ boundary and set $E=\mathbf{R}^{2} \backslash P$. If $P$ is strictly convex, the dual billiard $T: E \rightarrow E$ is defined as follows. For any point $o \in E$ there are two rays $R$ and $R^{\prime}$ emanating from $o$ and tangent to $P$, where the observer looking at $P$ from $o$ sees $R$ on the left and $R^{\prime}$ on the right of $P$. Let $A$ and $A^{\prime}$ be the points of tangency. For any point $v \in \mathbf{R}^{2}$ denote by $r_{v}$ the Euclidean reflection about $v$. Then $T(o)=r_{A}(o)$. The mapping $T$ is continuous, preserves the Lebesgue measure and invertible with $T^{-1}(o)=r_{A^{\prime}}(o)$.

If $P$ is not strictly convex (for instance, $P$ is a convex polygon) the dual billiard mapping $T$ is defined the same way but not on all of $E$ (Fig. 1). Denote by $\sigma_{1}$ the union of straight lines through the sides of $P$. Then both $T$ and $T^{-1}$ are defined on $E \backslash \sigma_{1}$ and $\sigma_{1} \cap E$ is the union of singular sets of $T$ and $T^{-1}$. By induction on $n \geqq 1$ we define $\sigma_{n}$, a finite union of straight lines, where $T^{k},-n \leqq k \leqq n$, are well defined on $E \backslash \sigma_{n}$. The singular set $\Sigma=\bigcup_{n=1}^{\infty} \sigma_{n}$ is a countable union of straight lines, and for $x \in E \backslash \Sigma$ (regular points) the infinite orbits $\left\{T^{n} x:-\infty<n<\infty\right\}$ are defined. The theme of this work is the orbit behavior for dual polygonal billiards. In particular, can they be unbounded? If $P$ is not a polygon but is bounded by a $C^{7}$-curve of positive curvature, all of the orbits are bounded [M1, D]. The proof is based on the

* Partially supported by NSF Grant DMS 88-02643

$\star \star$ Permanent address: Mathematical Institute of the Hungarian Academy of Sciences, P.O. Box 127, H-1364 Budapest, Hungary 
Fig. 1. Dual billiard about a polygon

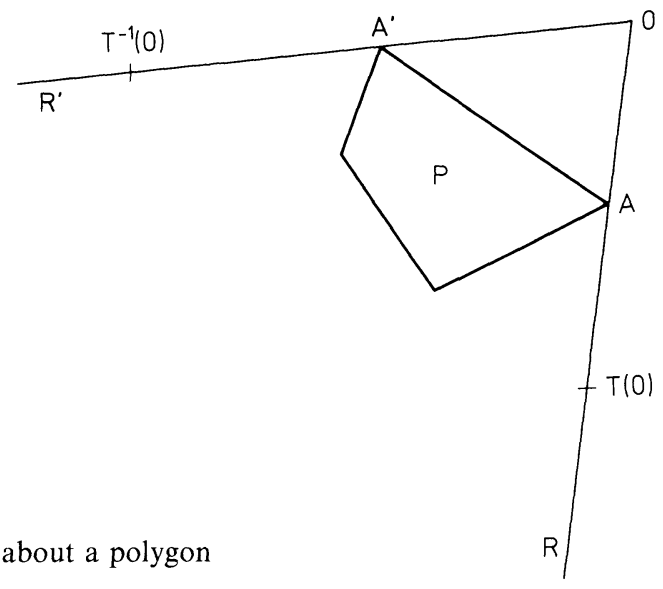

twist-map technique, thus the smoothness of the boundary curve of $P$ is essential. The question whether the dual billiard orbits are bounded if $P$ has corners, in particular if $P$ is a polygon, was formulated in [M2] and discussed in [VS, K].

The main claim of [VS and $\mathrm{K}$ ] is that if a polygon $P$ satisfies certain conditions then the orbits are bounded and under further assumptions on $P$ the orbits are periodic. We have tried hard to unravel the statements and the argument in [VS, K]. To no avail. The exposition in [K] seems to follow that of [VS], but is more cryptic, which makes it even worse. When we learned that the referee and the editor of [VS] had the same problem with it and could not help us, we gave up on $[\mathrm{VS}]$ and $[\mathrm{K}]$ and took up the problem on our own. The results are reported here. In the rest of the introduction we briefly describe the contents of the paper.

In Sect. 2 we introduce the notions of the necklace and the necklace dynamics. Let $o \in E$ and let $\left\{T^{n} o\right\}$ be the corresponding orbit. Imagine an observer located at $o$. From his point of view, $o$ is at rest and $P$ moves, getting reflected each time about one of its vertices. The successive positions of $P$ form a necklace $\left\{P_{n}\right\}$ with $P_{0}=P$ (Fig. 2). The necklaces about $O$ are the orbits of the corresponding necklace dynamics. The one-to-one correspondence between the orbits of the dual billiard and the necklaces (Proposition 2.2) allows to replace the study of the dual billiard map by the study of the necklace dynamics. This is analogous to the technique of reflecting the billiard table in polygonal billiards [G2].

Further in Sect. 2 we associate with any convex polygon $P$ and a point $o$ a polygonal line $Q$ unique up to dilations about $o$. The rationale for $Q$ is that any necklace about $o$, if it is sufficiently far from $o$, roughly follows $Q$. We prove (Proposition 2.5, the proof is in Sect. 3) that $Q$ is actually closed thus defining a polygon - the necklace polygon $Q$. The necklace polygon $Q(P)$ is determined by $P$ uniquely up to scaling and translations. Let $P$ have $p$ vertices and let $q \leqq p$ be the number of directions of the sides of $P$. Then $Q(P)$ is a centrally symmetric $2 q$-gon.

The necklace dynamics is given by a selfmapping $W$ of the set $\mathscr{P}$ of strongly regular polygons congruent to $P$ (see Sect. 2). In Sect. 4 we associate with each vertex of $Q(P)$ a subset of $\mathscr{P}$ which is naturally isomorphic to a disjoint union $S$ of two (truncated) semiinfinite strips (minus the singular set which is a countable collection of intervals). The induced first return map $F: S \rightarrow S$ is a local translation. More precisely, $S$ is tiled by a countable set of polygons $S_{i}$, and $F$ restricted to $S_{i}$ is 
the translation by a vector $v_{i}$. The singular set of $F$ is contained in $\bigcup b d\left(S_{i}\right)$, thus the union of the singular sets of the mappings $F^{n},-\infty<n<\infty$, is at most a countable union of intervals. Our original question becomes: are the (regular) orbits of $F$ bounded?

In order to answer this question, we study in Sect. 4 a slightly more general (than $F$ ) class of mappings: local translations of multiple truncated strips. A local translation $F: S \rightarrow S$ is periodic if there is a vector $\vec{p} \neq 0$ along $S$ such that for any $x \in S$ we have $F(x+\vec{p})=F(x)+\vec{p}$. Our main technical result (Theorem 4.6) says that if a local translation $F$ is invertible and periodic then the orbits of $F$ are bounded.

A polygon $P$ is called quasi-rational if its necklace polygon $Q(P)$ satisfies certain rationality conditions (Definition 3.2). A polygon $P$ is rational if its vertices belong to the integer lattice in some coordinate system on $\mathbf{R}^{2}$ (Definition 2.7). Any rational polygon is quasi-rational (Proposition 3.3) but not vice versa. For instance, the regular $n$-gons are quasi-rational but not rational for $n \neq 3,4,6$.

In Sect. 5 we apply Theorem 4.6 to the dual polygonal billiards. Theorem 5.1 says that if $P$ is quasi-rational then the dual billiard orbits are bounded. If $P$ is rational the orbits are periodic. A crucial point in the proof is that the first return map $F: S \rightarrow S$ corresponding to a quasi-rational polygon is periodic.

The technique of Sect. 4 allows to obtain the upper and lower bounds for the dual billiard trajectories. Denote by $|x|$ the distance from a point $x$ to the polygon $P$. By Theorem 5.3, there are positive constants $a<b, A$ and $B$ (depending only on $P$ ) such that for any $x$ and all $n$

$$
a|x|-A \leqq\left|T^{n}(x)\right| \leqq b|x|+B .
$$

Therefore, if $x$ is sufficiently far from $P$, the dual billiard orbit $\left\{T^{n}(x):-\infty\right.$ $<n<\infty\}$ is bounded away from $P$ (Corollary 5.3).

Let $P$ be a quasi-rational but not rational polygon. We expect the dual billiard about $P$ to have nonperiodic orbits. Our expectation seems to be confirmed by the dual billiard about the regular octagon [GS].

\section{Preliminaries}

Let $P$ be a convex polygon, $E=\mathbf{R}^{2} \backslash P$ and let $\sigma(P)$ be the union of straight lines through the sides of $P$. Any point $o \in E \backslash \sigma(P)$ (these points are called regular about $P$ ) uniquely determines a vertex $A_{1}$ of $P$ such that $T(o)=r_{A_{1}}(o)$ (we use notation of Sect. 1). Set $P_{1}=r_{A_{1}}(P)$. If $o \in E \backslash \sigma\left(P_{1}\right)$, it determines a vertex $A_{2}$ of $P_{1}$ and we set $P_{2}=r_{A_{2}}\left(P_{1}\right)$. After $n$ steps of this process we obtain a sequence $P_{0}=P, P_{1}, \ldots, P_{n}$ of polygons and a sequence $A_{1}, \ldots, A_{n}$ of their vertices (Fig. 2). Setting for brevity $r_{A_{i}}=r_{i}$ we have $P_{i+1}=r_{i+1}\left(P_{i}\right), 0 \leqq i \leqq n-1$.

2.1. We say that $\left\{P_{0}=P, P_{1}, \ldots, P_{n}\right\}$ is a necklace of length $n$ about $o$. It is obtained by developing $P$ ( $n$ times) about $o$ in the positive direction. Reversing the direction (which corresponds to replacing $T$ by $T^{-1}$ ) we get the necklace $\left\{P_{0}=P, P_{-1}, \ldots, P_{-m}\right\}$ obtained by developing $P$ ( $m$ times) about $o$ in the negative direction.

We can extend the necklace $\left\{P_{-m}, \ldots, P_{0}, \ldots, P_{n}\right\}$ indefinitely in both directions as long as $o$ is regular about $P_{k},-\infty<k<\infty$. We call such points strongly regular about $P$. Let $G$ be the group of reflections and translations of $\mathbf{R}^{2}$ and denote by $G_{P}$ 
Fig. 2. A necklace

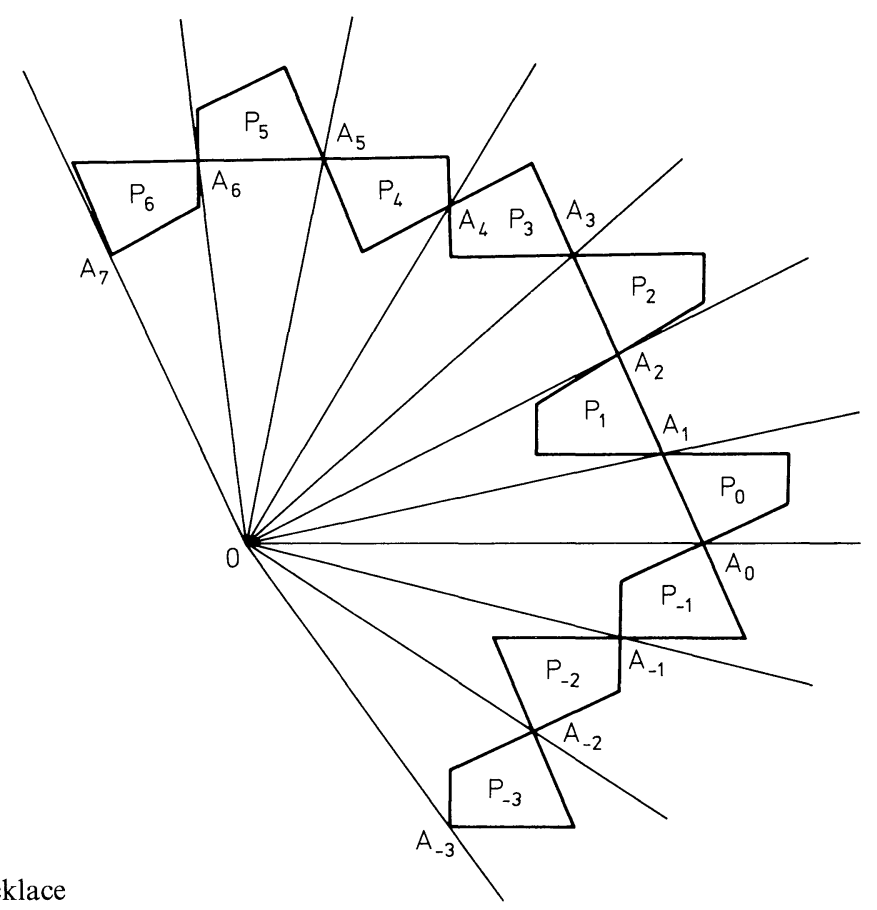

the subgroup generated by reflections about the vertices of $P$. The set $\Sigma(P)$ $=\left\{g \sigma(P): g \in G_{P}\right\}$ is a countable union of straight lines. The set $R_{P}$ of strongly regular points contains $E \backslash \Sigma(P)$.

Definition. Let $o \in R_{P}$ and let $N(P, o)=\left\{\ldots, P_{-m}, \ldots, P_{0}=P, \ldots, P_{n}, \ldots\right\}$ be the corresponding infinite necklace. We say that the necklace $N(P, o)$ is bounded if the set $\bigcup_{k=-\infty}^{k=\infty} P_{k}$ is bounded. We say that the necklace $N(P, o)$ is periodic if $P_{n}=P_{0}$ for some $n>0$. The definition of a bounded necklace applies in an obvious way to the semiinfinite necklaces

$$
N_{-}(P, o)=\left\{\ldots, P_{-m}, \ldots, P_{0}=P\right\} \quad \text { and } \quad N_{+}(P, o)=\left\{P_{0}=P, \ldots, P_{n}, \ldots\right\} .
$$

2.2. Proposition. 1. Let $-m \leqq 0 \leqq n$ and let $o \in E$. The finite necklace

$$
N_{-m}^{n}(P, o)=\left\{P_{-m}, \ldots, P_{0}=P, \ldots, P_{n}\right\}
$$

is defined simultaneously with the finite orbit $\left\{T^{k}(o),-m \leqq k \leqq n\right\}$. For $1 \leqq k \leqq n$, $-m \leqq-l \leqq-1$ we have

$$
\text { a) } T^{k}(o)=r_{1} \ldots r_{k}(o), \quad \text { b) } T^{-l}(o)=r_{-1} \ldots r_{-l}(o) \text {. }
$$

2. Let $o \in R_{P}$. The infinite orbit $\left\{T^{k}(o),-\infty<k<\infty\right\}$ is bounded if and only if the necklace $N(P, o)$ is bounded. The statement applies in an obvious way to the semiinfinite orbits and necklaces.

3. Let $o \in R_{P}$. The orbit $\left\{T^{k}(o),-\infty<k<\infty\right\}$ is periodic if and only if the necklace $N(P, o)$ is periodic. 
Proof. 1) Consider the necklace $\left\{P_{0}, \ldots, P_{n}\right\}$ and denote by $T_{k}$ the dual billiard about $P_{k}, 0 \leqq k \leqq n, T_{0}=T$. Set $o_{1}=T(o)$. The reflection $r_{1}$ sends $\left(o_{1}, P_{0}\right)$ into $\left(o, P_{1}\right)$ and conjugates $T$ with $T_{1}: T=r_{1} T_{1} r_{1}$ (Fig. 1).

It suffices to prove (1a) for $k=n$. We will prove it by induction on $n$. For $n=1$ it is trivial: $T(o)=r_{1}(o)$. For $n>1$,

$$
T^{n}(o)=T^{n-1}(T(o))=T^{n-1}\left(o_{1}\right)=\left(r_{1} T_{1}^{n-1} r_{1}\right)\left(o_{1}\right)=r_{1} T_{1}^{n-1}(o) .
$$

By definition, $\left\{P_{1}, \ldots, P_{n}\right\}$ is a necklace of length $n-1$ about $o$. Hence, by inductive assumption, $T_{1}^{n-1}(o)=r_{2} \ldots r_{n}(o)$, and (1a) follows. Equation (1b) is equivalent to (1a) under the substitution $T \rightarrow T^{-1}$. We leave the details to the reader.

2) and 3) Denote by $d(X, Y)$ the distance between the sets $X, Y \subset \mathbf{R}^{2}$. The orbit $\left\{T^{k}(o)\right\}$ is bounded if and only if the sequence $d\left(T^{k}(o), P\right)$ is bounded. By Eq. (1), $d\left(T^{k}(o), P\right)=d\left(o, P_{k}\right)$ which proves 2). Let $o$ be a periodic point, $T^{n}(o)=o$, and let $\left\{P_{0}, \ldots, P_{n}\right\}$ be the corresponding necklace with $P_{n}=r_{n} \ldots r_{1}\left(P_{0}\right)$. By Eq. (1), $r_{n} \ldots r_{1}(o)=o$, thus either $r_{n} \ldots r_{1}$ is the identity or it is the reflection about $o(n$ has to be odd). In the first case $P_{n}=P_{0}$ and the necklace $\left\{P_{0}, \ldots, P_{n}\right\}$ is periodic. In the second case the reflection $r_{o}$ provides an isomorphism of $\left(o, P_{0}\right)$ and $\left(o, P_{n}\right)$. Therefore $r_{o}$ induces an isomorphism of the necklaces $\left\{P_{0}, \ldots, P_{n}\right\}$ and $\left\{P_{n}, \ldots, P_{2 n}\right\}$, hence $P_{2 n}=P_{0}$ and the necklace $\left\{P_{0}, \ldots, P_{2 n}\right\}$ is periodic. The proof of the converse is even easier and we leave it to the reader.

2.3. Fix an "origin" $o \in \mathbf{R}^{2}$ and let $P$ be a convex $n$-gon not containing $o$. We say that $P$ is in a regular position (about $o$ ) if $o \notin \sigma(P)$. Let $l_{1}, \ldots, l_{m}$ be the straight lines through $o$ parallel to the sides of $P(m=n$ if $P$ does not have parallel sides). The lines $l_{1}, \ldots, l_{m}$ divide $\mathbf{R}^{2}$ into $2 m$ closed cones $C_{1}, \ldots, C_{2 m}$ and we denote by $R_{1}, \ldots, R_{2 m}$ their boundary rays enumerated counterclockwise (Fig. 3). In what follows we use the convention that $C_{2 m+1}=C_{1}, R_{2 m+1}=R_{1}$. We have $-C_{i}=C_{i+m},-R_{i}=R_{i+m}$.

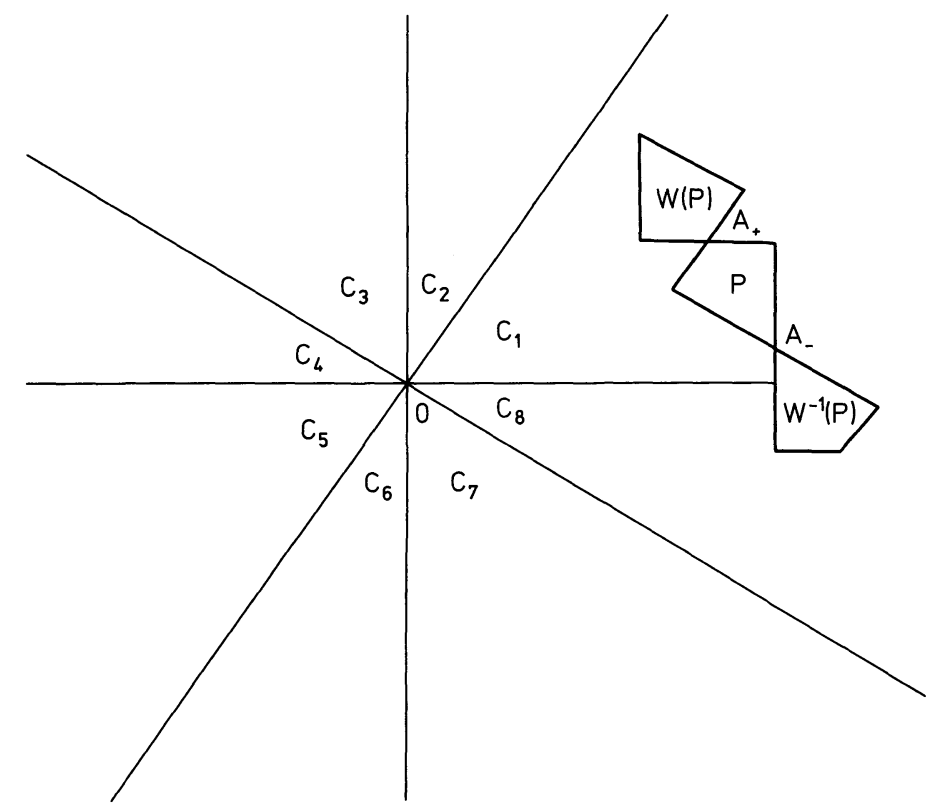

Fig. 3. The system of cones corresponding to a polygon and a point; the head and the tail of $P$; the necklace mapping 
Let us denote our original polygon by $P_{0}$ and consider the set $\mathscr{R}$ of polygons $P$ congruent to $P_{0}$ (i.e. $P=g P_{0}, g \in G$ ) and in a regular position about $o$. Notice that the preceding construction does not depend on the choice of $P$ in $\mathscr{R}$. For any such $P$ let $T$ be the dual billiard map about $P$ and let $A_{+}, A_{-}$be the vertices of $P$ such that $T(o)=r_{A_{+}}(o), T^{-1}(o)=r_{A_{-}}(o)$ (Fig. 3). We call $A_{+}$the head of $P, A_{-}$the tail of $P$ and denote the corresponding reflections by $r_{+}, r_{-}$. In the necklace $\left\{r_{-}(P), P, r_{+}(P)\right\}$ (Fig. 3) the polygons $r_{-}(P), r_{+}(P)$ are congruent to $P_{0}$ and do not contain $o$. Denote the set of such polygons by $\mathscr{S}$ and define the mapping $W: \mathscr{R} \rightarrow \mathscr{S}$ by $W(P)=r_{+}(P)$. Analogously, we set $W^{-1}(P)=r_{-}(P)$.

We define the set $\mathscr{P} \subset \mathscr{R}$ of polygons $P$ strongly regular about o by requiring that $W^{n}(P) \in \mathscr{R}$ for all $n$. The construction above defines an invertible selfmapping $W$ of $\mathscr{P}$ with the inverse $W^{-1}$. The complement $\mathscr{R} \backslash \mathscr{P}$ is analogous to the singular set $\Sigma(P)$ of the dual billiard mapping and like $\Sigma(P)$ has codimension one. We call $W$ the necklace mapping. By Proposition 2.2, the correspondence between $T$ and $W$ preserves the periodic and the bounded orbits. This allows us to forget (temporarily) the mapping $T$ and study the orbits of $W$, i.e. the necklace dynamics.

If $A, B$ are points in $\mathbf{R}^{2}$, we denote by $[A B]$ the closed segment joining them and by $A B$ the corresponding vector. We also indicate vectors by upper arrows, e.g. $\vec{a}$, and use notation $A+\vec{a}$ for the usual action of vectors on $\mathbf{R}^{2}$, e.g. $A+A B=B$.

2.4. Choose a cone $C$ from the set $\left\{C_{i}: 1 \leqq i \leqq 2 m\right\}$ and let $R_{+}, R_{-}$be its boundary rays (Fig. 4). Denote by $\mathscr{S}_{C}\left(\mathscr{R}_{C}, \mathscr{P}_{C}\right)$ the subset of $\mathscr{S}(\mathscr{R}, \mathscr{P})$ consisting of polygons $P \subset C$.

Lemma - Definition. Let $P \in \mathscr{R}_{C}$ and let $A_{+}, A_{-}$be the head and the tail of $P$. The vector $A_{-} A_{+}$does not depend on the choice of $P \in \mathscr{R}_{C}$. We denote this vector by $\vec{a}_{C}$ and call it the necklace vector corresponding to the cone $C$.

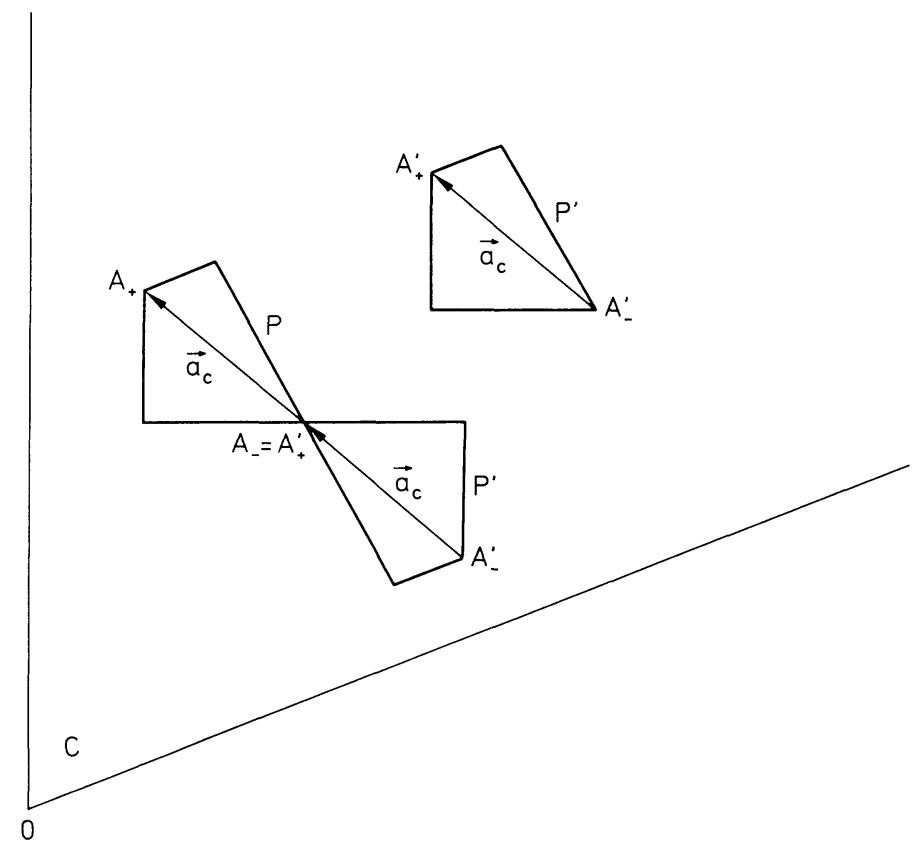

Fig. 4. The necklace vector 
Proof. Let $P^{\prime} \in \mathscr{R}_{C}$ be another polygon and let $A_{+}^{\prime}, A_{-}^{\prime}$ be the head and the tail of $P^{\prime}$. Suppose first that $P^{\prime}$ differs from $P$ by a translation: $P^{\prime}=P+\vec{a}$. Then $A_{+}^{\prime}=A_{+}+\vec{a}$, $A_{-}^{\prime}=A_{-}+\vec{a}$ (Fig. 4), hence $A_{-}^{\prime} A_{+}^{\prime}=A_{-} A_{+}$. If $P^{\prime}$ differs from $P$ by a reflection, $P^{\prime}=r P$, we can assume that $r$ is the reflection about $A_{-}$(Fig. 4). Then $A_{+}^{\prime}=A_{-}$, $A_{-}^{\prime}=r\left(A_{+}\right)$and $A_{-}^{\prime} A_{+}^{\prime}=r\left(A_{+} A_{-}\right)=A_{-} A_{+}$.

Corollary. We have $\vec{a}_{-C}=-\vec{a}_{C}$.

Proof. Let $P \in \mathscr{R}_{C}$ and let $\vec{a}_{C}=A_{-} A_{+}$. Calculating $\vec{a}_{-C}$ using the polygon $r_{0} P \in \mathscr{R}_{-C}$ we obtain the assertion.

2.5. We denote by $\vec{a}_{i}$ the necklace vectors corresponding to the cones $C_{i}, 1 \leqq i \leqq 2 m$. Choose a point $A_{1} \in R_{1}\left(A_{1} \neq o\right)$ and draw the ray emanating from $A_{1}$ in the direction of $\vec{a}_{1}$ until it crosses $R_{2}$ at a point $A_{2}$. Repeat this construction until we come back to the ray $R_{1}=R_{2 m+1}$ obtaining in the process the polygonal line $Q=A_{1} A_{2} \ldots A_{2 m+1}$. If we make another choice $A_{1}^{\prime} \in R_{1}$, where $o A_{1}^{\prime}=\lambda\left(o A_{1}\right), \lambda>0$, we obtain the similar polygonal line

$$
Q^{\prime}=A_{1}^{\prime} A_{2}^{\prime} \ldots A_{2 m+1}^{\prime}=\lambda\left(A_{1} A_{2} \ldots A_{2 m+1}\right) \text {. }
$$

Proposition - Definition. We have $A_{2 m+1}=A_{1}$ thus $Q=A_{1} A_{2} \ldots A_{2 m+1}$ is a closed polygon. Any change of the data involved in the definition of $Q$ (i.e. $o, A_{1} \in R_{1}$ and the choice of $R_{1}$ ) changes $Q$ by translations and dilations only. Thus the polygon $Q=Q(P)$ is determined by $P$ uniquely up to translations and dilations, and we call it the necklace polygon of $P$. The necklace polygon of $P$ is a convex centrally symmetric $2 m-g o n$.

We postpone the proof of the proposition until Sect. 3.

2.6. The dual billiards are naturally covariant with respect to the group $H$ of orientation preserving affine transformations of $\mathbf{R}^{2}$. More precisely, let $P$ and $P^{\prime}$ be two convex polygons. Denote by $T$ and $T^{\prime}$ the corresponding dual billiard mappings of the respective regions $E$ and $E^{\prime}$. If $P^{\prime}=h P, h \in H$, then $h: E \rightarrow E^{\prime}$ is an isomorphism and $T^{\prime}=h T h^{-1}$.

In view of the above, the theory of dual billiards should be $H$-covariant. A subset $L \subset \mathbf{R}^{2}$ is called a lattice if $L=h \mathbf{Z}^{2}$, where $h \in H$ and $\mathbf{Z}^{2}$ is the standard integer lattice.

Lemma. Let $P$ be an arbitrary polygon and let $G_{P}$ be the corresponding group. The group $G_{P}$ is discrete if and only if the vertices of $P$ belong to a lattice.

Proof. Both properties are $H$-invariant. Suppose first that the vertices of $P$ belong to a lattice $L$. Acting by $H$, if necessary, we can assume that $L=\mathbf{Z}^{2}$. The group $G_{P}$ is contained in the group generated by reflections about the points of $\mathbf{Z}^{2}$, which is discrete, hence $G_{P}$ is discrete. Take this as an assumption now and let $A_{1}, \ldots, A_{n}$ be the vertices of $P$. Set $\vec{a}_{i}=2 A_{1} A_{i}, 2 \leqq i \leqq n$. The group $L$ generated by the translations $\vec{a}_{i}, 2 \leqq i \leqq n$, is contained in $G_{P}$, hence it is discrete. Therefore $L$ is a lattice.

2.7. Definition. A polygon $P$ is called rational if the group $G_{P}$ is discrete or, equivalently, if the vertices of $P$ belong to a lattice.

Remarks. 1. By definition, the set of rational polygons is $H$-invariant. All triangles are rational. For $n>3$ rational $n$-gons are dense in the set of all $n$-gons (in the natural topology). 2. The reader should not confuse the notion of rational polygon in the present context with its counterpart in the theory of polygonal billiards (see, e.g., [G1, Definition 6]). The two notions are dual to each other. 


\section{Necklace Polygon}

Let $N(P, o)=\left\{\ldots, P_{-m}, \ldots, P_{0}=P, \ldots, P_{n}, \ldots\right\}$ be a necklace (finite or infinite) and let

$$
\left\{\ldots, V_{-m}, \ldots, V_{0}, \ldots, V_{n+1}, \ldots\right\}
$$

be the corresponding sequence of heads and tails, where $V_{i}$ is the tail of $P_{i}$ and the head of $P_{i-1}$. The (finite or infinite) polygonal line

$$
\Gamma=\left[\ldots, V_{-m}, \ldots, V_{0}, \ldots, V_{n+1}, \ldots\right]
$$

is the string of the necklace $N(P, o)$.

3.1. Proof of Proposition 2.5. Assume the opposite, i.e. that $A_{2 m+1} \neq A_{1}$ and call the piecewise linear curve $\left[A_{1} \ldots A_{2 m+1}\right]$ the necklace polygonal line. Replacing $T$ by $T^{-1}$, if necessary, we can assume without loss of generality that $\left|o A_{2 m+1}\right|<\left|o A_{1}\right|$ (Fig. 5). The ratio

$$
0<\left|o A_{2 m+1}\right| /\left|o A_{1}\right|=\mu<1
$$

does not depend on the position of $A_{1}$ on the ray $R=R_{1}$. Hence starting the necklace polygonal line $S$ at any point $A \in R$ and turning around $o$ once in the positive direction, we return to $R$ at the point $A^{\prime}$ where $o A^{\prime}=\mu(o A)$. Continuing $S$ indefinitely we obtain a selfsimilar infinite polygonal spiral about $o$ that spirals into $o$ with the rate $1 / \mu$.

Let $N(P, o)$ be an infinite necklace with $P_{0} \in \mathscr{P}$ intersecting the ray $R$ and let $\Gamma$ be the corresponding infinite string. Consider the necklace polygonal line $S_{0}$ starting at the point $A=\left[V_{0} V_{1}\right] \cap R$ and returning to $R$ at $A_{1}$. Follow the string $\Gamma$ from the point $A$ counterclockwise until it returns to $R$, and denote by $A_{1}^{\prime}$ the point of return. We take the distance $\left|A_{1} A_{1}^{\prime}\right|$ for the measure of the deviation between $S_{0}$ and $\Gamma$ after one turn around $o$.

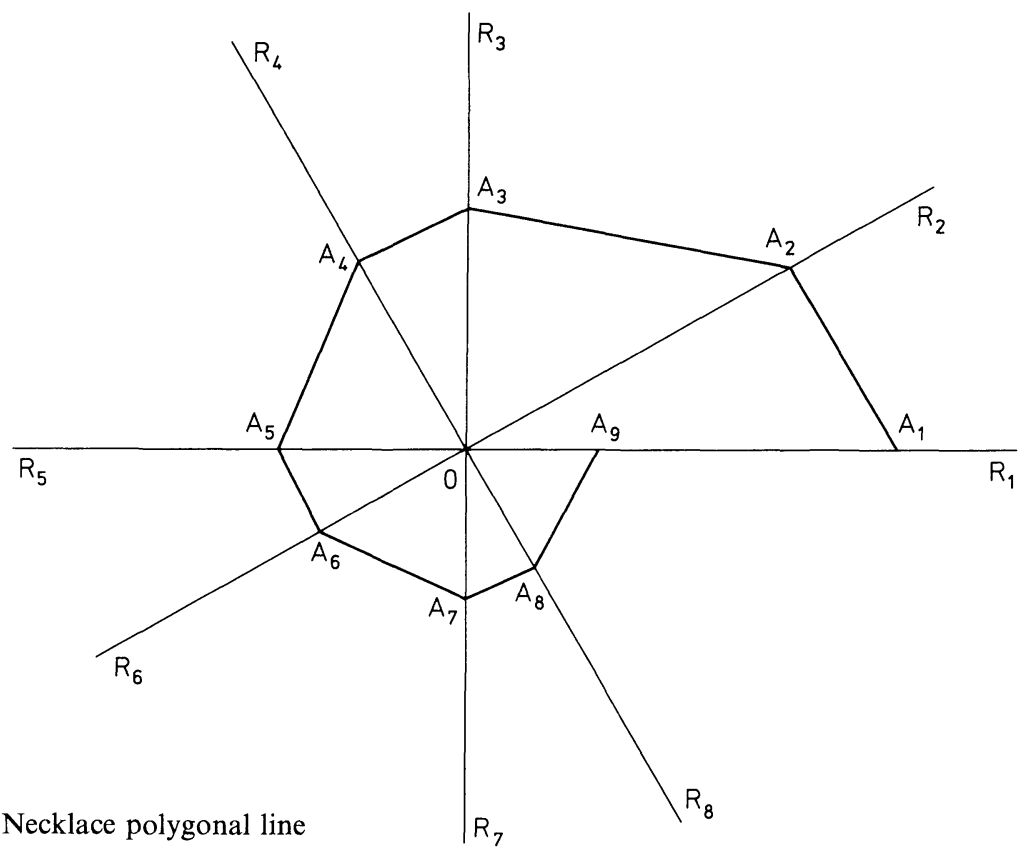

Fig. 5. Necklace polygonal line 
Recall the system of cones $C_{1}, \ldots, C_{2 m}$ we have introduced in Sect. 2. By Lemma 2.4, a segment $\left[V_{i} V_{i+1}\right]$ of $\Gamma$ located inside a cone $C$ of the system is parallel to the corresponding necklace vector $\vec{a}_{C}$. Therefore the deviation between $\Gamma$ and $S_{0}$ can increase only at the crossings with the rays $R_{1}, \ldots, R_{2 m+1}$. Denote by $d$ the diameter of $P$. There is a constant $a$ depending only on $P$ modulo dilations such that at each crossing the deviation increases by at most $a d$. Thus after one turn around $o$ the deviation between $\Gamma$ and $S_{0}$ is at most $(2 m+1) a d=c$, where $c$ does not depend on the starting point $A$. We have

$$
\left|o A_{1}^{\prime}\right| \leqq\left|o A_{1}\right|+\left|A_{1} A_{1}^{\prime}\right| \leqq \mu|o A|+c .
$$

Let $S_{1}$ be the necklace polygonal line starting at $A_{1}^{\prime}$ and going around $o$ once in the positive direction, and let $A_{2}$ be the point where it comes back to $R$. Follow $\Gamma$ from $A_{1}^{\prime}$ in the positive direction until it crosses $R$ and let $A_{2}^{\prime}$ be the crossing point. Continuing by induction, we obtain two sequences $\left\{A_{n}\right\}$ and $\left\{A_{n}^{\prime}\right\}$ of points in $R$ $\left(n \geqq 0, A_{0}=A_{0}^{\prime}=A\right)$.

The preceding argument shows that for all $n$ we have $\left|A_{n} A_{n}^{\prime}\right| \leqq c$. Hence, by construction and Eq. (2)

$$
\left|o A_{n+1}^{\prime}\right| \leqq\left|o A_{n+1}\right|+\left|A_{n+1} A_{n+1}^{\prime}\right| \leqq \mu\left|o A_{n}^{\prime}\right|+c .
$$

It follows by induction from Eq. (3) that

$$
\left|o A_{n}^{\prime}\right| \leqq \mu^{n}|o A|+(1-\mu)^{-1} c .
$$

We reverse the direction and follow the string $\Gamma$ clockwise. Repeating the preceding construction we obtain the sequences $\left\{A_{-n}\right\}$ and $\left\{A_{-n}^{\prime}\right\}, n \geqq 0$, of points on $R$. The argument of Eq. (3) applies and yields

$$
\left|o A_{-(n+1)}^{\prime}\right| \geqq \mu^{-1}\left|o A_{-n}^{\prime}\right|-c .
$$

Set $v=\mu^{-1}>1$. From Eq. (5), by induction on $n$, we obtain that if $|o A|>c(v-1)^{-1}$, we have for all $n>0$,

$$
\left|o A^{\prime}{ }_{-n}\right| \geqq v^{n}(|o A|-c /(v-1))+c /(v-1) .
$$

By Eq. (4), the polygons $P_{k}, k>0$, stay within a finite distance from the origin. By Eq. (6), the distance from $P_{-k}$ to the origin grows exponentially as $k \rightarrow \infty$ (at least if $P_{0}$ is sufficiently far from $o$ ).

Suppose now that the polygon $P_{0}$ (and therefore all $P \in \mathscr{P}$ ) is rational. By Lemma 2.6, the set of polygons $\left\{P_{k}:-\infty<k<\infty\right\}$ is discrete. The set $\left\{P_{k}: 0<k\right\}$, being discrete and bounded, is finite, i.e. the necklace $N(P, o)$ is periodic. This contradicts to the earlier conclusion that the set $\left\{\bigcup P_{-k}: k \geqq 0\right\}$ is unbounded. Thus Proposition 2.5 holds for rational polygons $P$.

The necklace polygonal line $Q$ depends not only on $P$ but on the choice of "origin" $o$, the ray $R$ (from the system of rays parallel to the sides of $P$ ) and a point $A$ on $R$. Let us fix this data for $P$ (assume for simplicity that $P$ has no parallel sides). Consider the polygons $P^{\prime}$ (with the same number of sides) which are sufficiently close to $P$. For any such $P^{\prime}$ there is a unique ray $R^{\prime}$ (from $o$ ) close to $R$ and a unique point $A^{\prime}$ on $R^{\prime}$ such that $\left|o A^{\prime}\right|=|o A|$. The polygonal line $Q^{\prime}$ uniquely determined by this data is close to $Q$. In other words (with an obvious normalisation), $Q(P)$ continuously depends on $P$.

Let now $P$ be an arbitrary polygon and let $P_{i}$ be a sequence of rational polygons converging to $P$. Since $Q_{i}=Q\left(P_{i}\right)$ are closed polygons and since $Q_{i} \rightarrow Q(P)$ as $i \rightarrow \infty$, $Q(P)$ is a closed polygon. 
Corollary. The necklace polygon $Q(P)$ is a convex centrally symmetric polygon determined by $P$ uniquely up to translations and dilations.

Proof. Consider the necklace polygon $Q$ as a polygonal line $Q=A_{1} \ldots A_{2 m+1}$ determined (in addition to $P$ ) by $o, R$ and a point $A_{1} \in R$. Varying $A_{1}$ on $R$ changes $Q$ by dilations about $o$. Choosing another ray $R^{\prime}$ corresponds to a change of $A_{1}$ on $R$, i.e. it produces only dilations. Taking another point $o^{\prime}$ for the origin corresponds to a parallel translation of the system of rays $\left\{R_{1}, \ldots, R_{2 m}\right\}$, thus it produces a translation of $Q$.

Since the convexity of $Q$ is obvious from construction, it remains to show that $Q$ is symmetric about $o$. By Corollary 2.4, the triangles $\Delta_{i}=A_{i} o A_{i+1}$ and $\Delta_{m+i}=A_{m+i} O A_{m+i+1}$ are similar for all $i$. Denote by $r_{i}>0$ the corresponding dilation coefficient: $\Delta_{m+i}=-\mathrm{r}_{i} \Delta_{i}$ (the minus corresponds to the symmetry about $o$ ). Comparing the adjacent triangles we see that $r_{i}$ does not depend on $i:\left|o A_{m+i}\right|$ $=r\left|o A_{i}\right|$. Therefore $\left|o A_{2 m+1}\right|=r^{2}\left|o A_{1}\right|$, and, by Proposition $2.5, r=1$.

3.2. Let $P$ be a convex polygon and let $C_{i}, 1 \leqq i \leqq 2 m$, be the corresponding system of cones about a point $o$ with the necklace vectors $\vec{a}_{i}$. Let $Q=A_{1} \ldots A_{2 m+1}$ be a necklace polygon of $P$ centered at $o$. By Proposition 2.5, there are $2 m$ positive numbers $r_{i}$ such that

$$
A_{i} A_{i+1}=r_{i} \vec{a}_{i}, \quad 1 \leqq i \leqq 2 m
$$

and $r_{m+i}=r_{i}$. By Corollary 3.1, the numbers $r_{i}$ are determined by $P$ uniquely up to a common factor, thus the point $\left(r_{1}: \ldots: r_{m}\right) \in \mathbf{R P}^{m-1}$ (the real projective space of dimension $m-1)$ is determined by $P$ uniquely up to a cyclic permutation.

Definition. A polygon $P$ is called quasi-rational if the numbers $r_{1}, \ldots, r_{m}$ are rational (up to a common factor), i.e. $\left(r_{1}: \ldots: r_{m}\right) \in \mathbf{Q} \mathbf{P}^{m-1}$.

Lemma. Let $P$ be a quasi-rational polygon. There exists a necklace polygon $Q=A_{1} \ldots A_{2 m+1}$ such that

$$
A_{i} A_{i+1}=k_{i} \vec{a}_{i}, \quad 1 \leqq i \leqq 2 m,
$$

where $k_{i}$ are positive integers.

Proof. Let $Q$ be an arbitrary necklace polygon corresponding to $P$, and let $o$ be the center of $Q$. Let $r_{i}=r_{i}(Q), 1 \leqq i \leqq m$, be the numbers in Eq. (7) corresponding to $Q$. For the necklace polygon $\lambda \bar{Q}$ obtained from $Q$ by dilation by $\lambda>0$, we have $r_{i}(\lambda Q)$ $=\lambda r_{i}(Q), 1 \leqq i \leqq m$. Since $P$ is quasi-rational, there is $\lambda_{1}>0$ such that $r_{i}^{\prime}=r_{i}\left(\lambda_{1} Q\right)$ are rational numbers. Let $\lambda_{2}$ be the least common denominator of $r_{i}^{\prime}, 1 \leqq i \leqq m$. Then the numbers $r_{i}\left(\lambda_{1} \lambda_{2} Q\right)=\lambda_{1} \lambda_{2} r_{i}=k_{i}$ are integers. Thus the necklace polygon $\lambda_{1} \lambda_{2} Q$ satisfies (8).

3.3. Proposition. Any rational polygon is quasi-rational.

Proof. Let $(x, y)$ be a coordinate system on $\mathbf{R}^{2}$ and let $o=(0,0)$. A point $A \in \mathbf{R}^{2}$ is called rational if it has rational coordinates. A vector $\vec{a}$ is rational if $\vec{a}=o A$ and $A$ is a rational point. A line in $\mathbf{R}^{2}$ is rational if it contains two rational points.

Let $P$ be a rational polygon and let $(x, y)$ be a coordinate system on $\mathbf{R}^{2}$ such that the vertices of $P$ are rational points and $o=(0,0)$. Then the rays $R_{i}$ (corresponding to $P$ and $o$ ) define rational lines and the necklace vectors $\vec{a}_{i}$ are rational. Choose a rational point $A_{1}$ on $R_{1}$ and let $Q=A_{1} \ldots A_{2 m+1}$ be the corresponding necklace 
polygon. The line containing $A_{1}$ and parallel to $\vec{a}_{1}$ is rational, hence $A_{2}$, as a point of intersection of rational lines, is rational. Continuing by induction, we conclude that all vertices of $Q$ are rational points, hence the vectors $A_{i} A_{i+1}$ are rational. Two collinear rational vectors differ by a rational scalar, hence the numbers $r_{i}$ in Eq. (7) are rational.

3.4. Remark. Let $P$ be a regular $n$-gon and let $Q$ be the corresponding necklace polygon. If $n$ is even then $Q$ is a regular $n$-gon, if $n$ is odd then $Q$ is a regular $2 n$-gon. In any case, the numbers $r_{i}$ of Eq. (7) are all equal, hence the regular $n$-gon is quasirational for all $n$. On the other hand, it is rational only for $n=3,4,6$. Thus rational polygons form a proper subset of the set of quasi-rational polygons.

\section{Necklace Dynamics}

We use notation of Sect. 2 and assume that $P_{0}$ is not centrally symmetric (unless specified otherwise). Then $\mathscr{S}=\mathscr{S}_{+} \cup \mathscr{S}_{-}$, a disjoint union, where $P=P_{0}+\vec{a}$ for $P \in \mathscr{S}_{+}$and $P=r_{A}\left(P_{0}\right)$ for $P \in \mathscr{S}_{-}\left(\vec{a}, r_{A}\right.$ are uniquely determined by $\left.P\right)$. We choose a cone $C$ from our system of cones (see Fig. 3), denote by $C^{\prime}$ the following cone, by $R^{\prime}$ the ray separating $C$ from $C^{\prime}$, and let $R$ be the other boundary ray of $C$. We denote by $\mathscr{I}_{R}$ the set of $P$ in $\mathscr{S}$ intersecting $R$ and set

$$
\mathscr{S}_{ \pm}^{R}=\mathscr{S}_{ \pm} \cap \mathscr{S}_{R}, \quad \mathscr{R}_{ \pm}^{R}=\mathscr{S}_{ \pm}^{R} \cap \mathscr{R}, \quad \mathscr{P}_{ \pm}^{R}=\mathscr{S}_{ \pm}^{R} \cap \mathscr{P} .
$$

4.1. Let $P_{1}, P_{2} \in \mathscr{S}_{+}^{R}\left(\right.$ or $\left.\mathscr{S}_{-}^{R}\right)$ and assume that $P_{i} \cap C^{\prime}=\emptyset, i=1,2$. Assume that the heads $A_{1}, A_{2}$ of $P_{1}, P_{2}$ are well defined (e.g. $P_{i} \in \mathscr{R}_{+}^{R}$ ). By the argument of Lemma 2.4, $A_{2}=A_{1}+\vec{t}$, where $\vec{t}$ is the unique vector such that $P_{2}=P_{1}+\vec{t}$. Therefore the correspondence $P \rightarrow A$ which assigns to every $P \in \mathscr{R}_{ \pm}^{R}, P \cap C^{\prime}=\emptyset$, its head $A$, uniquely extends to a mapping $h: \mathscr{S}_{+}^{\mathbf{R}} \cup \mathscr{S}_{-}^{\boldsymbol{R}} \rightarrow \mathbf{R}^{2}$ satisfying $h(P+\vec{t})$ $=h(P)+\vec{t}$. We set $h\left(\mathscr{S}_{ \pm}^{R}\right)=S_{ \pm}$(suppressing the dependence on $R$ ). By construction, both mappings $h: \mathscr{S}_{ \pm}^{\underline{R}} \rightarrow S_{ \pm} \subset \mathbf{R}^{2}$ are one-to-one.

Figure 6 introduces the notion of a truncated strip. It shows an infinite strip divided by a finite polygonal line into two truncated strips. The boundary of a truncated strip $S$ consists of two parallel rays $R$ and $\widetilde{R}$ and a polygonal line $L$. A vector like the vector $\vec{t}$ on Fig. 6 (i.e., parallel to $R$ and pointing to $\infty$ ) is said to be along the truncated strip $S$.

Lemma. The sets $S_{+}, S_{-}$are truncated strips. The lower boundary ray of each is $R$ while the upper boundary ray is contained in $R+\vec{a}_{C}$.

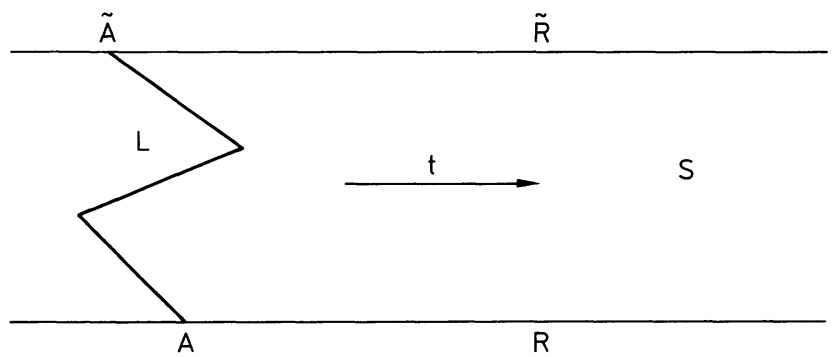

Fig. 6. Truncated strip 
Proof. Consider, for concreteness, the polygons $P$ in $\mathscr{S}_{+}^{R}$. Denote by $A=A(P)$ and $B=B(P)$ the "head" and the "tail" of $P$. By our convention, $A(P)(B(P))$ is indeed the head (tail) of $P$ if $P \subset \operatorname{Int}(C)$. Otherwise, for any $P^{\prime} \subset \operatorname{Int}(C), P^{\prime}=P+\vec{t}$, we have $A\left(P^{\prime}\right)$ $=A(P)+\vec{t}, B\left(P^{\prime}\right)=B(P)+\vec{t}$. If $A$ belongs to a side $s$ of $P$ parallel to $R$ then $A$ is closer to $o$ than the other vertex of $s$ (Fig. 3). Therefore, for $P \in \mathscr{S}_{+}^{R}$ with $A(P) \in R$ the points $A(P)$ sweep all of $R$ forming the lower part of the boundary of $S_{+}$. For $P$ in $\mathscr{S}_{+}^{\boldsymbol{R}}$ with $B(P) \in R$ the points $B(P)$ sweep a subray $\varrho \subset R(\varrho \neq R$ if $B$ belongs to a side $S$ parallel to $R$ ). The corresponding points $A(P)$ span the ray $\widetilde{R}=\varrho+\vec{a}_{C}$ (see Lemma 2.4) which is the upper part of the boundary of $S_{+}$. The remaining part of the boundary of $S_{+}$is swept by the points $A(P)$, where $o \in b d(P)$, i.e. it is a finite polygonal line.

4.2. We consider the cone $C$ with the boundary rays $R, R^{\prime}$ and the corresponding truncated strips $S_{+}, S_{-}$. For $n \geqq 0$ we define the ray $R_{n}^{\prime}=R^{\prime}-n \vec{a}$, where $\vec{a}=\vec{a}_{C}$ is the necklace vector. Let $R_{n}^{\prime}$ intersect $R(\widetilde{R})$ at $D_{n}\left(E_{n}\right)$, and for $n>0$ denote by $\pi_{n}$ the parallelogram $D_{n-1} E_{n-1} E_{n} D_{n}$ (Fig. 7). We think of $S_{+}$and $S_{-}$as being located on two different copies of $\mathbf{R}^{2}$ and denote by $\pi_{n}^{+}, \pi_{n}^{-}$the corresponding copies of $\pi_{n}$ $(n>o)$. Then $S_{+}\left(S_{-}\right)$is the union of $\pi_{n}^{+},\left(\pi_{n}^{-}\right), n>0$, and the closed polygon $\pi_{0}^{+}\left(\pi_{0}^{-}\right)$. For the case shown in Fig. 7, $\pi_{0}^{+}$is the "truncated triangle" $o E_{0} \tilde{o} K o$. Denote by $\vec{d}, \vec{b}$ the vectors $D_{0} D_{1}, D_{0} E_{0}$. By Lemma 4.1 , for $n>0$, the vectors $D_{n} E_{n-1}$ are equal to the necklace vector $\vec{a}$, and $\pi_{n}^{ \pm}+\vec{d}=\pi_{n+1}^{ \pm}$(Fig. 7).

Remark. The constructions above are well defined only on polygons in regular position. Therefore the constructions below will be well defined only on a set of regular points in $S_{+}\left(S_{-}\right)$. To simplify the exposition, in what follows we ignore the singular set which is a countable union of intervals in $S_{+}\left(S_{-}\right)$. This should not cause any confusion.

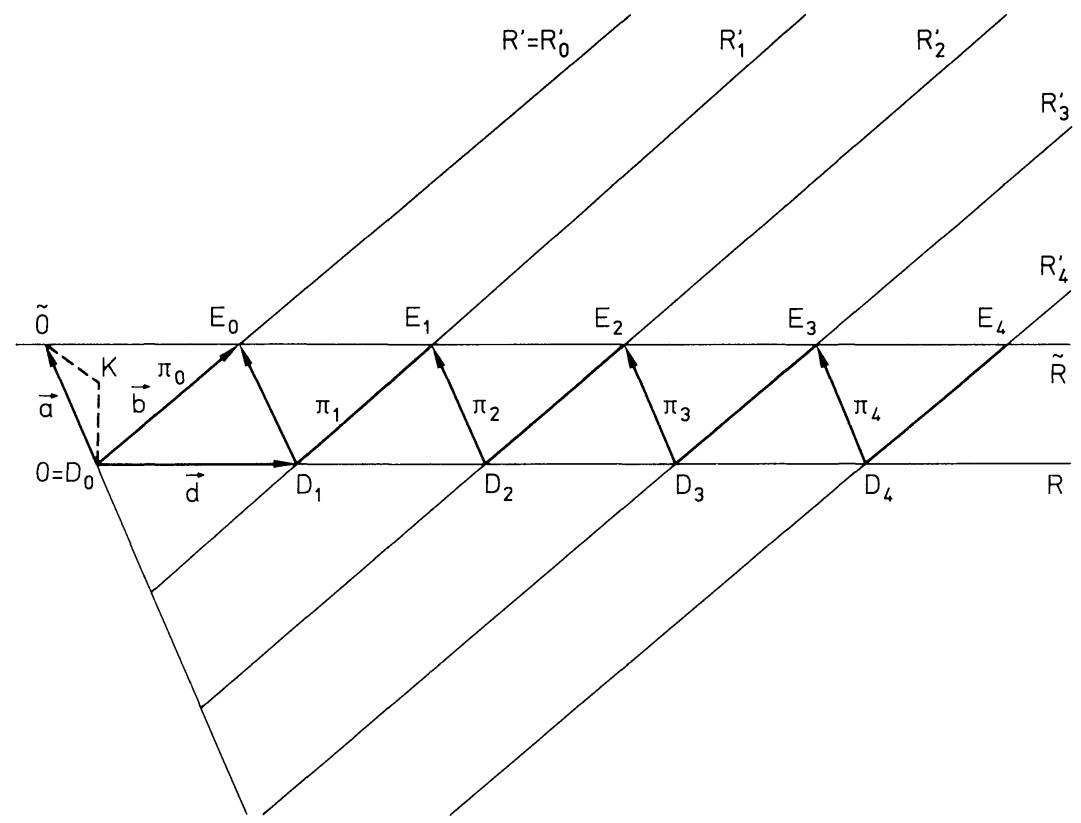

Fig. 7. Decomposition of truncated strip 
Let $S=S_{+} \cup S_{-}$be the "abstract" disjoint union (the double truncated strip). We use "prime" to denote the counterparts of the objects above corresponding to the ray $R^{\prime}$, e.g. $S_{ \pm}^{\prime}$ are the truncated strips with the base $R^{\prime}$. For $P \in \mathscr{S}_{R}$ there is a unique $k=k(P) \geqq 0$ such that $W^{k}(P) \in \mathscr{S}_{R^{\prime}}$. This defines a mapping $P \rightarrow W^{k}(P)$ of $\mathscr{S}_{R}$ into $\mathscr{S}_{R^{\prime}}$. Under the isomorphisms $h: \mathscr{S}_{R} \rightarrow S, h^{\prime}: \mathscr{S}_{R^{\prime}} \rightarrow S^{\prime}$ it becomes a mapping $f: S \rightarrow S^{\prime}$ of the double truncated strips.

Lemma. There exists a vector-valued function $\vec{\tau}=\vec{\tau}( \pm, n \bmod 2)$ such that for $A \in \pi_{n}^{ \pm}$ $(n \geqq 0)$ we have

$$
f(A)=A+n \vec{a}+\vec{\tau} .
$$

Proof. Let $\sigma_{n}(n \geqq 1)$ be the strip between the rays $R_{n-1}^{\prime}$ and $R_{n}^{\prime}$ (Fig. 7). Then $\pi_{n}^{ \pm}=\sigma_{n} \cap S_{ \pm}$. The head of a polygon $P$ belongs to $\sigma_{n}$ if (and only if) $W^{n}(P)$ intersects $R^{\prime}$. Let $A \in \pi_{n}$ be the head of $P$ and let $\left\{P, P_{1}, \ldots, P_{n}=P^{\prime}\right\}$ be the corresponding necklace (Fig. 8). Set $A_{k}=A+k \vec{a}, 1 \leqq k \leqq n$. By Lemma 2.4, for $k<n, A_{k}$ is the head of $P_{k}=W^{k}(P)$, and $A_{n}$ is a vertex of $P^{\prime}$. The "intrinsic" position of the head $A^{\prime}$ of $P^{\prime}$ is determined by the "pairity" $\operatorname{sgn}\left(P^{\prime}\right)$ of $P^{\prime}$, where $\operatorname{sgn}\left(P^{\prime}\right)= \pm$ for $P^{\prime} \in \mathscr{S}_{R^{\prime}}^{ \pm}$. An analogous statement holds for the head $A$ of $P$, hence for the vertex $A_{n}=A+n \vec{a}$ of $P^{\prime}$. Thus the "correction vector" $\vec{\tau}=A_{n} A^{\prime}$ depends only on the pair $\operatorname{sgn}(P), \operatorname{sgn}\left(P^{\prime}\right)$. Since $\operatorname{sgn}\left(P^{\prime}\right)=\operatorname{sgn}(P)(-1)^{n}$, Eq. (9) holds for $n \geqq 1$. Our convention for the vertices $h(P), h^{\prime}\left(P^{\prime}\right)$ if $P \in \pi_{0}, \pi_{0}^{\prime}$ automatically extends Eq. (9) to the case $n=0$.

Remark. An elaboration of the argument above shows that the "head correction vector" $\vec{\tau}$ can take at most two values.

The isomorphisms $h, h^{\prime}$ transfer the functions $\operatorname{sgn}(P), \operatorname{sgn}\left(P^{\prime}\right)$ from $\mathscr{S}_{R}, \mathscr{S}_{R^{\prime}}$ to the double strips $S, S^{\prime}$. We denote the transferred functions by $\operatorname{sgn}(A), \operatorname{sgn}\left(A^{\prime}\right)$.

Corollary. The mapping $f: S \rightarrow S^{\prime}$ satisfies

$$
f(A+2 \vec{d})=f(A)+2 \vec{b} .
$$

In particular, $\operatorname{sgn}(f(A+2 \vec{d}))=\operatorname{sgn}(f(A))$.

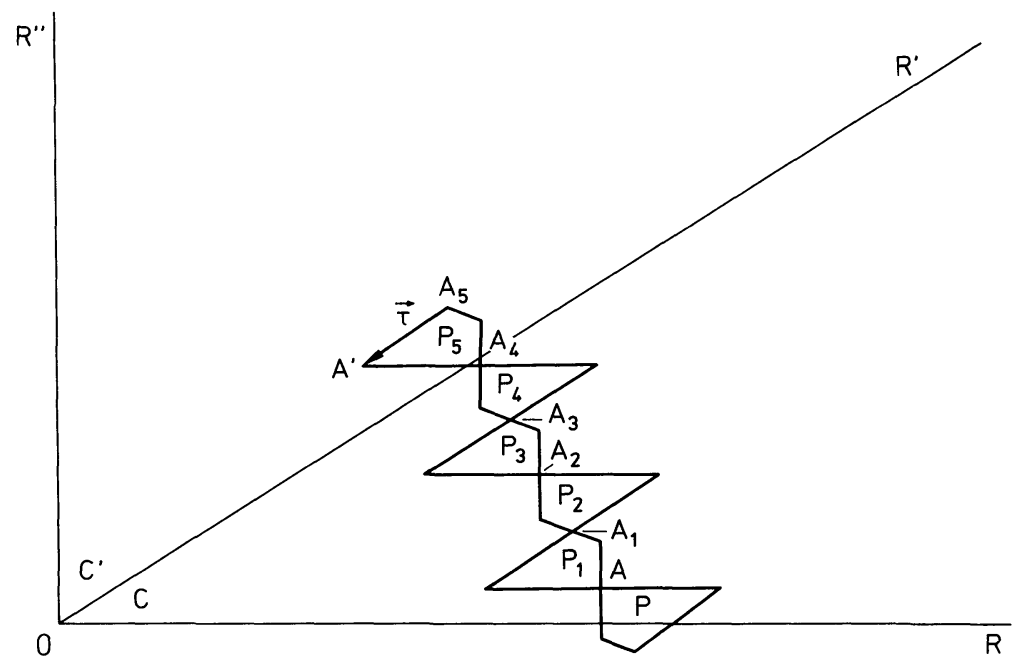

Fig. 8. The mapping $f: S \rightarrow S^{\prime}$ 
Proof. The vector $\vec{d}(\vec{b})$ is along the strip $S\left(S^{\prime}\right)$. The translation $A \rightarrow A+2 \vec{d}$ sends $\pi_{n}^{ \pm}$ into $\pi_{n+2}^{ \pm}$. Let $A \in \pi_{n}$ and let $f(A)=A+n \vec{a}+\vec{\tau}$. Then $A+2 \vec{d} \in \pi_{n+2}$ and, by Eq. (9)

$$
f(A+2 \vec{d})=A+2 \vec{d}+(n+2) \vec{a}+\vec{\tau}=f(A)+2(\vec{a}+\vec{d})=f(A)+2 \vec{b} .
$$

4.3. Let $D$ be a plane domain with a decomposition $D=\bigcup P_{i}, i \in I$, where $P_{i}$ are subdomains with piecewise smooth boundaries $\partial P_{i}$ (e.g. polygons), and, for $i \neq j$, $P_{i} \cap P_{j} \subset \partial P_{i} \cap \partial P_{j}$. A mapping $f: D \rightarrow D^{\prime}$ (possibly singular on $\bigcup \partial P_{i}$ ) is called a local translation with respect to the decomposition $D=\bigcup_{i \in I} P_{i}$ if there is a vector valued function $\vec{t}$ on $I$ such that for $x \in P_{i}$

$$
f(x)=x+\vec{t}_{i} .
$$

The definition naturally extends to the situation when $D, D^{\prime}$ are abstract disjoint unions of (a finite number of) plane domains, e.g., $D=D_{+} \cup D_{-}, D^{\prime}=D_{+}^{\prime} \cup D_{-}^{\prime}$, and the domains $D_{ \pm}=\bigcup P_{i}^{ \pm}$satisfy the conditions above. Denote by $\varepsilon=\{ \pm\}$ the index set for $D$ and by $\varepsilon^{\prime}=\{ \pm\}$ that for $D^{\prime}$. A local translation $f: D \rightarrow D^{\prime}$ is given by a vector valued function $\vec{t}(\varepsilon, i)$ and by an index-valued function $\varepsilon^{\prime}(\varepsilon, i)$ so that for $x \in P_{i}^{\varepsilon}$ we have

$$
f(x)=x+\vec{t}(\varepsilon, i), \quad f(x) \in D_{\varepsilon^{\prime}(\varepsilon, i)}^{\prime} .
$$

A mapping $f: D \rightarrow D^{\prime}$ from an abstract union of plain domains into another one is a local translation if it can be put into the form above.

Let $f: D \rightarrow D^{\prime}, f^{\prime}: D^{\prime} \rightarrow D^{\prime \prime}$ be local translations. Then the composition $f^{\prime} f: D \rightarrow D^{\prime \prime}$ is a local translation.

Proposition. We use notation of Subsect.4.2. The mapping $f: S \rightarrow S^{\prime}$ is a local translation with respect to the decomposition $S_{ \pm}=\bigcup_{n>0} \pi_{n}^{ \pm}$. The vector valued function $\vec{t}$ on $\{ \pm\} \times\{n \geqq 0\}$ is given by $\vec{t}(\varepsilon, n)=n \vec{a}+\vec{\tau}(\varepsilon, n \bmod 2)$. The $\{ \pm\}$-valued function $\varepsilon^{\prime}$ is given by $\varepsilon^{\prime}(\varepsilon, n)=\varepsilon(-1)^{n}$.

Proof. Follows immediately from Lemma 4.2.

4.4. We return to the system of cones $\left\{C_{1}, \ldots, C_{2 m}\right\}$ and rays $\left\{R_{1}, \ldots, R_{2 m}\right\}$ we have associated with our dual polygonal billiard. With every cone $C_{i}$ (bounded by the rays $R_{i}, R_{i+1}$ ) we associate (as in Sects. 4.1, 4.2) the double truncated strip $S_{i}=S_{i}^{+} \cup S_{i}^{-}$, the decomposition $S_{i}^{ \pm}=\bigcup_{n>0} \pi_{n}^{ \pm}(i)$ and the vectors $\vec{a}_{i}, \vec{b}_{i}, \vec{a}_{i}(i=1, \ldots, 2 m$ with the convention $2 m+1=1$ ). By Proposition 4.3 , the mappings $f_{i}: S_{i} \rightarrow S_{i+1}$ are local translations, hence the product $F=f_{2 m} \ldots f_{1}$ is a local translation of $S=S_{1}$ into itself. By construction, $F$ is the first return map associated with $W$. More precisely, for $x \in S$ there is a unique polygon $P$ (intersecting $R=R_{1}$ ) such that $x$ is the head of $P$. We follow the necklace of $P:\left\{P_{k}=W^{k}(P), k>0\right\}$ until it returns to $R$. Let it return on a polygon $P^{\prime}=P_{n}$ and let $x^{\prime}$ be the head of $P^{\prime}$. Then $x^{\prime}=F(x)$. Set $\vec{d}=\vec{d}_{1}$ (a vector along $S$ ). Define the function $\varepsilon(x)$ on $S$ with values in $\{ \pm\}$ by $\varepsilon^{\prime}=\varepsilon(x) \varepsilon$. Let $x \in S_{\varepsilon}$ and let $F(x) \in S_{\varepsilon^{\prime}}\left(\varepsilon, \varepsilon^{\prime}= \pm\right)$.

Proposition. Let the polygon $P$ defining the dual billiard be quasi-rational. Then there exists a positive integer $n$ such that $F$ satisfies

$$
F(x+2 n \vec{d})=F(x)+2 n \vec{d} .
$$

Equation (12) implicitly means that $\varepsilon(x+2 n \vec{d})=\varepsilon(x)$. 
Proof. We use the preceding notation and denote by $\Delta_{i}$ the triangle with the side vectors $\vec{a}_{i}, \vec{d}_{i}, \vec{b}_{i}(i=1, \ldots, 2 m)$ and vertex $o$. Let $Q=A_{1} \ldots A_{2 m}$ be an arbitrary necklace polygon of $P$ and let $A_{i} A_{i+1}=r_{i} \vec{a}_{i}, r_{i}>0,1 \leqq i \leqq 2 m$. The polygon $Q$ is divided into $2 m$ triangles $o A_{i} A_{i+1}$, where $o A_{i} A_{i+1}=r_{i} \Delta_{i}$ (similarity of triangles). Therefore, for $1 \leqq i \leqq 2 m$,

$$
o A_{i+1}=r_{i} \vec{b}_{i}=r_{i+1} \vec{d}_{i+1} .
$$

Suppose now that the polygon $P$ is quasi-rational. By Lemma 3.2, we can assume that the numbers $r_{i}$ are integers, $r_{i}=n_{i}, 1 \leqq i \leqq 2 m$. Applying Corollary 4.2 to the mappings $f_{i}: S_{i} \rightarrow S_{i+1}$ and using Eq. (13), we obtain that for any $x \in S_{i}$,

$$
f_{i}\left(x+2 n_{i} \vec{d}_{i}\right)=f_{i}(x)+2 n_{i} \vec{b}_{i}=f_{i}(x)+2 n_{i+1} \vec{d}_{i+1} .
$$

Iterating Eq. (14) for $i=1, \ldots, 2 m$ we obtain that for $x \in S$,

$$
\left(f_{2 m} \ldots f_{1}\right)\left(x+2 n_{1} \vec{d}_{1}\right)=\left(f_{2 m} \ldots f_{1}\right)(x)+2 n_{1} \vec{d}_{1} \text {. }
$$

This proves the proposition with $n=n_{1}$.

Denote the vector $2 n \vec{d}$ by $\vec{p}$. For any $x \in S$ and $k>0$ the point $x+k \vec{p} \in S$ while $x-k \vec{p}$ may not belong to $S$.

Corollary. Let $k \in \mathbf{Z}$ and $x \in S$. If $x+k \vec{p} \in S$ then

$$
F(x+k \vec{p})=F(x)+k \vec{p} .
$$

Proof. Reduce to the case $k>0$ and apply Eq. (12).

4.5. Set $\Pi_{0}^{ \pm}=\pi_{1}^{ \pm} \cup \ldots \cup \pi_{2 n}^{ \pm}, \Pi_{1}^{ \pm}=\pi_{2 n+1}^{ \pm} \cup \ldots \cup \pi_{4 n}^{ \pm}$, etc. ... Also set $\Pi_{-1}^{ \pm}=\pi_{0}^{ \pm}$. The "double" parallelogram $\Pi=\Pi_{0}^{+} \cup \Pi_{0}^{-}$is a "fundamental domain" of $S$ with respect to the translations by $k \vec{p}$, i.e., for any $x \in S$ there is a unique $x_{0} \in \Pi$ and a unique integer $v(x) \geqq-1$ such that

$$
x=x_{0}+v(x) \vec{p},
$$

where $v(x)=k$ for $x \in \Pi_{k}$. Note that in general not all points of the form $x_{0}-\vec{p}$ belong to $S$ because $\Pi_{-1}^{ \pm}$are truncated parallelograms.

Lemma-Definition. We define a mapping $\Phi: \Pi \rightarrow \Pi$ and an integer valued function $\tau(x)$ on $\Pi$ by the equation

$$
F(x)=\Phi(x)+\tau(x) \vec{p},
$$

where $\tau(x)=v(F(x)), \Phi(x) \in \Pi$. The pair $(\Phi, \tau)$ uniquely determines $F$. The mapping $\Phi$ is invertible and $\Phi, \Phi^{-1}: \Pi \rightarrow \Pi$ are local translations.

Proof. Equation (17) is obtained by applying (16) to the point $F(x), x \in \Pi$. By Corollary 4.4 and Eq. (16), for any $x \in S$

$$
F(x)=\Phi\left(x_{0}\right)+\tau\left(x_{0}\right) \vec{p}+v(x) \vec{p}
$$

which uniquely determines $F$. The mapping $F$ is invertible by definition. The inverse mapping $F^{-1}: S \rightarrow S$ is obtained by reversing the directions of the necklaces in the constructions of Subsects. 4.3, 4.4. Hence $F^{-1}$ is a local translation and satisfies the periodicity condition (15). Applying Eq. (17) to $F^{-1}$ we obtain a mapping $\Phi^{\prime}: \Pi \rightarrow \Pi$ and an integer valued function $\tau^{\prime}$ on $\Pi$ where

$$
F^{-1}(x)=\Phi^{\prime}(x)+\tau^{\prime}(x) \vec{p} .
$$


From Eqs. (17)-(19) we see that $\Phi^{\prime}=\Phi^{-1}, \tau^{\prime}(x)=-\tau\left(\Phi^{-1}(x)\right)$, hence

$$
F^{-1}(x)=\Phi^{-1}(x)-\tau\left(\Phi^{-1}(x)\right) \vec{p} .
$$

Since $F$ is a local translation, the function $\tau$ is locally constant and Eq. (17) implies that $\Phi$ is a local translation. The same argument applies to $\Phi^{-1}$.

Corollary. Equation (17) establishes a one-to-one correspondence between the mappings $F: S \rightarrow S$ and the pairs $(\Phi: \Pi \rightarrow \Pi, \tau: \Pi \rightarrow\{n \geqq-1\})$. The function $\tau$ corresponding to an invertible mapping $F$ can take values -1,0,1 only.

Proof. The first assertion is immediate from the proof of Lemma 4.5. If $F$ is invertible we have the function $\tau^{\prime}$ defined by Eq. (19) with values $\geqq-1$. By Eq. (20), $\tau(x)=-\tau^{\prime}(y) \leqq 1$, hence the only possible values are 0 and \pm 1 .

4.6. The notion of a double truncated strip $S=S_{+} \cup S_{-}$has an obvious generalisation. Namely, let $S=\bigcup_{\varepsilon \in E} S_{\varepsilon},|E|<\infty$, be an abstract disjoint union of a finite number of truncated strips with a vector $\vec{p}$ which is along all $S_{\varepsilon}$. We think of $S$ as a subset of $|E|$ copies of $\mathbf{R}^{2}$ and say that $S$ is a multiple truncated strip. The setting of Subsect. 4.5 immediately extends to the selfmappings of multiple truncated strips. Leaving the details to the reader, we will use the notation and the results of Subsect. 4.5 in this slightly more general situation.

Theorem. Let $S$ be a multiple truncated strip and let $F: S \rightarrow S$ be a local translation satisfying the periodicity condition (15). 1. If $F$ is invertible, then the orbits $\left\{F^{k}(x):-\infty<k<\infty\right\}$ are bounded. 2. Let $F$ be invertible and assume that the translation vectors $\vec{t}(\varepsilon, i)$ defining $F$ generate a discrete group. Then the orbits of $F$ are periodic.

Proof. 1. Let $\Pi=\bigcup_{\varepsilon \in E} \Pi_{\varepsilon}, \Pi \subset S$, be the "multiple parallelogram" associated with $F$ and let $(\Phi, \tau)$ be the data corresponding to $F$. Here $\Phi: \Pi \rightarrow \Pi$ is a local translation and $\tau: \Pi \rightarrow \mathbf{Z}$ is an integer valued function with $\tau(x) \geqq-1$. For any $n \geqq 1$ the mapping $F^{n}: S \rightarrow S$ satisfies Eq. (15). From Eqs. (17) and (18) we obtain that the data $\left(\Phi_{n}, \tau_{n}\right)$ corresponding to $F^{n}$ are given by $\Phi_{n}=\Phi^{n}: \Pi \rightarrow \Pi$ and

$$
\tau_{n}(x)=\tau(x)+\tau(\Phi x)+\ldots+\tau\left(\Phi^{n-1} x\right) .
$$

Let $\left(\Phi^{-1}, \tau_{-1}\right)$ correspond to the inverse mapping $F^{-1}: S \rightarrow S$ and let $\left(\Phi^{-n}, \tau_{-n}\right)$ be the data for $F^{-n}, n \geqq 1$, where, by Eq. (20),

$$
\tau_{-n}(x)=\tau_{-1}(x)+\tau_{-1}\left(\Phi^{-1} x\right)+\ldots+\tau_{-1}\left(\Phi^{-(n-1)} x\right)=-\tau_{n}\left(\Phi^{-n} x\right) .
$$

By definition of $\tau_{k}$ [Eq. (17)], we have for $x \in \Pi,-\infty<k<\infty$,

$$
F^{k}(x)=\Phi^{k}(x)+\tau_{k}(x) \vec{p},
$$

therefore an orbit $\left\{F^{k}(x):-\infty<k<\infty, x \in \Pi\right\}$ is bounded if and only if $\left|\tau_{k}(x)\right|$ $<$ const for all $k$. By Corollary 4.5 , for any $n \geqq 1$, the functions $\tau_{n}, \tau_{-n}$ take values \pm 1 and 0 only, hence for $x \in \Pi$,

$$
\left|\tau_{k}(x)\right| \leqq 1, \quad-\infty<k<\infty .
$$

We have shown that the orbits $\left\{F^{k}(x):-\infty<k<\infty\right\}$ are bounded for $x \in \Pi$. Equation (15) implies that they are bounded for all $x \in S$. 
2. Denote by $T$ the group generated by the translation vectors $\vec{t}(\varepsilon, i)$. By Eq. (11), for any $x \in S, n \in \mathbf{Z}$, there is $\vec{t}(x, n) \in T$ such that $F^{n}(x)=x+\vec{t}(x, n)$. If $T$ is a discrete group then any orbit $\left\{F^{n}(x):-\infty<n<\infty\right\} \subset\{x+T\}$ is discrete. Thus the orbits are bounded and discrete, hence finite.

\section{Main Results}

We return to the dual billiards and their orbits.

5.1. Theorem. Let $T$ be the dual billiard mapping about a polygon $P$. If $P$ is quasirational then the orbits of $T$ are bounded. If $P$ is rational the orbits of $T$ are periodic.

Proof. Let $o$ be a strongly regular point about $P$, i.e. the orbit

$$
O=\left\{T^{n}(o):-\infty<n<\infty\right\}
$$

is well defined. By Proposition 2.2, $O$ is bounded (periodic) if and only if the corresponding necklace $N(P, o)$ is bounded (periodic).

The necklace $N(P, o)$ is an orbit of the necklace dynamics mapping $W$. We use notation of Sect. 4. Let $S$ be the double truncated strip corresponding to a cone $C$ and let $F: S \rightarrow S$ be the induced mapping. It is clear from the construction of $F$ in Sect. 4 that an orbit of $W$ is bounded (periodic) if and only if the corresponding orbit of $F$ is bounded (periodic).

Let $P$ be quasi-rational. Then, by Proposition 4.4 and Theorem 4.6(1), the orbits of $F$ are bounded, hence, by the preceding argument, the dual billiard orbits are bounded. Let $P$ be rational. Since, by Proposition 3.3, $P$ is quasi-rational, the dual billiard orbits are bounded. By definition of rationality (Definition 2.7), the orbits are discrete, hence they are finite, i.e. periodic.

5.2. In the setting of Theorem 5.1 we want to estimate the spread of a dual billiard orbit. We will need a lemma about the necklace dynamics.

We use notation of Sect. 4 for a quasi-rational polygon $P$. Let $R$ and $R^{\prime}$ be arbitrary rays from the system of rays associated with $P$ (and a point $o$ ) and let $S, S^{\prime}$ be the corresponding double truncated strips. Let $S=\bigcup_{n \geqq-1} \Pi_{n}, S^{\prime}=\bigcup_{n \geqq-1} \Pi_{n}^{\prime}$ be the respective decompositions of the strips into the double parallelograms $\left(\Pi_{-1}^{-}, \Pi_{-1}^{\prime}\right.$ are truncated). Let $\vec{p}$ and $\vec{p}^{\prime}$ be the vectors along $S$ and $S^{\prime}$ such that (for $\left.n \geqq 0\right) \Pi_{n}+\vec{p}$ $=\Pi_{n+1}, \Pi_{n}^{\prime}+\vec{p}^{\prime}=\Pi_{n+1}^{\prime}$. With any integer $r$ we associate a mapping $\vec{f}: S \rightarrow S^{\prime}$ as follows. Let $x \in S$ and let $P(x)$ be the polygon with the head $x$. We develop $P(x)$ into a necklace (in the positive direction if $r \geqq 0$ and negative otherwise). We let the necklace make $|r|$ full turns about $o$, then we continue until it reaches $R^{\prime}$. Let $P^{\prime}$ be the corresponding polygon of the necklace and let $x^{\prime} \in S^{\prime}$ be its head. We set $x^{\prime}=f(x)$. Note that the mapping $F$ of Theorem 4.6 is the special case corresponding to $S^{\prime}=S, r=1$.

Lemma. Let $S, S^{\prime}, r$ be arbitrary and let $f: S \rightarrow S^{\prime}$ be the mapping defined above. For all $n \geqq-1$ we have

$$
f\left(\Pi_{n}\right) \subset \Pi_{n-1}^{\prime} \cup \Pi_{n}^{\prime} \cup \Pi_{n+1}^{\prime} .
$$

Proof. The argument of Sect. 4 applies and shows that $f$ corresponds to a mapping $\phi: \Pi \rightarrow \Pi^{\prime}$ and an integer valued function $\tau$ on $\Pi$ such that for $x \in \Pi$ (identified with $\left.\Pi_{0}\right)$

$$
f(x)=\phi(x)+\tau(x) \vec{p}^{\prime}
$$


and $\tau(x) \geqq-1$. The inverse mapping $f^{-1}: S^{\prime} \rightarrow S$ corresponds to $\phi^{-1}: \Pi^{\prime} \rightarrow \Pi$ and the function $\tau^{\prime}\left(x^{\prime}\right)=-\tau\left(\phi^{-1}\left(x^{\prime}\right)\right)$. Therefore $-1 \leqq \tau(x) \leqq 1$. The periodicity property

$$
f(x+n \vec{p})=f(x)+n \vec{p}^{\prime}
$$

implies the assertion.

Corollary. We use the preceding notation. For $x \in S$ denote by $X \subset \mathbf{R}^{2}$ the orbit of $x$ under the necklace dynamics. Let $x \in \Pi_{n}$. Then for any double strip $S^{\prime}$,

$$
X \cap S^{\prime} \subset \Pi_{n-1}^{\prime} \cup \Pi_{n}^{\prime} \cup \Pi_{n+1}^{\prime} \text {. }
$$

Proof. Immediate from the preceding lemma.

5.3. Denote by $d(x, P)$ the distance from $x \in E$ to $P$.

Theorem. Let $P$ be a quasi-rational polygon and let $T$ be the dual billiard about $P$. There are positive constants $a<b$ and $A, B$ such that for any (strongly regular) point $o$ and all $n$

$$
a \cdot d(o, P)-A \leqq d\left(T^{n}(o), P\right) \leqq b \cdot d(o, P)+B .
$$

Proof. Consider the infinite necklace

$$
N(P, o)=\left\{\ldots, P_{-m}, \ldots, P_{0}=P, \ldots, P_{n}, \ldots\right\}
$$

generated by $P$. By the proof of Proposition 2.2, Eq. (26) is equivalent to

$$
a \cdot d\left(P_{0}, o\right)-A \leqq d\left(P_{i}, o\right) \leqq b \cdot d\left(P_{0}, o\right)+B .
$$

In what follows we denote by const any positive constant if its value is irrelevant for the proof. Denote by $x_{i} \in E$ the head of $P_{i}$ and set $\|x\|=d(o, x)$. Since $\left|\left\|x_{i}\right\|-d\left(P_{i}, o\right)\right| \leqq \operatorname{diam}(P)$, Eq. (27) is equivalent to

$$
a\left\|x_{0}\right\|-\mathrm{const} \leqq\left\|x_{i}\right\| \leqq b\left\|x_{0}\right\|+\text { const } .
$$

Suppose that $x_{0}$ does not belong to any (double truncated) strip $S$. Then $P_{0} \subset C$ for some cone $C$. Denote by $x_{0}^{\prime}$ the head of the polygon $P_{l}$ in the necklace $N(P, o)$ such that $P_{l}$ crosses a boundary ray of $C$ and $|l|$ is the smallest possible. Then

$$
x_{0}^{\prime}=x_{0}+l \vec{a}+\vec{\tau}
$$

where $\vec{a}=\vec{a}_{C}$ and $\vec{\tau}$ is a "head correction vector." By elementary geometry, there exist constants $0<\alpha<\beta$ depending only on $P$ such that

$$
\alpha\left\|x_{0}\right\|-\operatorname{diam}(P) \leqq\left\|x_{0}^{\prime}\right\| \leqq \beta\left\|x_{0}\right\|+\operatorname{diam}(P) .
$$

Therefore, Eq. (28) is equivalent to the inequalities

$$
\text { const }\left\|x_{0}^{\prime}\right\|-\text { const } \leqq\left\|x_{i}\right\| \leqq \text { const }\left\|x_{0}^{\prime}\right\|+\text { const . }
$$

Equivalently, it suffices to show (28) under the assumption that $x_{0} \in S$ for some $S$.

Let $Q_{0}$ be the unique necklace polygon (about $o$ ) satisfying Eq. (8), where the maximal common divisor of the integers $k_{i}$ is equal to one. Let $R$ be the base ray of $S$ and let $\vec{a}, \vec{d}, \vec{b}$ be the corresponding vectors (Fig. 7). Denote by $k=k(S)$ the integer $k_{i}$ in Eq. (8) corresponding to $S$.

Any point $x \in S$ belongs to a unique parallelogram $\pi_{n}$, where $n=n(x)$. Then (see Fig. 7)

$$
\|x\| /\|d\|-\text { const } \leqq n(x) \leqq\|x\| /\|d\|+\text { const } .
$$


Let now $N(x)$ be the index $N$ such that $x \in \Pi_{N}$. Since $\Pi$ is $2 k$ times longer than $\pi$, we have, analogously to the preceding inequality

$$
\|x\| / 2 k\|d\|-\text { const } \leqq N(x) \leqq\|x\| / 2 k\|d\|+\text { const } .
$$

Let $N=N\left(x_{0}\right)$. By Corollary 5.2, the orbit $\left\{x_{i}:-\infty<i<\infty\right\}$ belongs to any polygonal annulus $A\left(\lambda_{1}, \lambda_{2}\right)=\left\{\bigcup \lambda Q_{0}: \lambda_{1} \leqq \lambda \leqq \lambda_{2}\right\}$ that contains $\Pi_{N-1} \cup \Pi_{N} \cup \Pi_{N+1}$. It suffices to take

$$
\lambda_{1}=(N-1)-c_{1}, \quad \lambda_{2}=(N+1)+c_{2},
$$

where the positive constants $c_{1}, c_{2}$ depend only on $P$. Combining this with (29) we obtain that the orbit $\left\{x_{i}:-\infty<i<\infty\right\}$ is covered by $A\left(\lambda_{1}, \lambda_{2}\right)$ with

$$
\left\|x_{0}\right\| / 2 k\|d\|-\text { const }<\lambda_{1}<\lambda_{2}<\left\|x_{0}\right\| / 2 k\|d\|+\text { const . }
$$

Denote by $\varrho_{1}\left(\varrho_{2}\right)$ the radius of the maximal (minimal) circle about $o$ inscribed into $Q_{0}$ (superscribed about $Q_{0}$ ). The polygonal annulus $A\left(\lambda_{1}, \lambda_{2}\right)$ is contained in the (usual) annulus about $o$ bounded by the circles with the radii $\lambda_{1} \varrho_{1}<\lambda_{2} \varrho_{2}$. In view of Eq. (30), we get

$$
\varrho_{1}\left\|x_{0}\right\| / 2 k\|d\|-\text { const }<\left\|x_{i}\right\|<\varrho_{2}\left\|x_{0}\right\| / 2 k\|d\|+\text { const }
$$

which finishes the proof.

Corollary. If a point $x$ is sufficiently far from $P$, its dual billiard orbit is bounded away from $P$.

Proof. Immediate from Eq. (26).

5.4. The following assertion has been proved in the course of proof of Theorem 5.3.

Corollary. Let $P$ be quasi-rational and let o be an arbitrary (strongly regular) point. Let $Q=\lambda Q_{0}$ be a necklace polygon (about $o$ ) intersecting $P$. There are $c_{1}, c_{2}>0$ (depending only on $P$ ) such that the necklace $N(P, o)$ is contained in the polygonal annulus

$$
A\left(\lambda-c_{1}, \lambda-c_{2}\right)=\left\{\bigcup \mu Q_{0}: \lambda-c_{1}<\mu<\lambda-c_{2}\right\} .
$$

\section{References}

[D] Douady, R.: Thèse de 3-ème cycle, Université de Paris 7, 1982

[G1] Gutkin, E.: Billiards on almost integrable polyhedral surfaces. Ergod. Theor. Dynam. Sys. 4, 529-584 (1984)

[G2] Gutkin, E.: Billiards in polygons. Physica 19D, 311-333 (1986)

[GS] Gutkin, E., Simanyi, N.: Dual billiard about the regular octagon (in preparation)

[K] Kołodziej, R.: The antibilliard outside a polygon. Bull. Polish Acad. Sci. Math. 37, 163-168 (1989)

[M1] J. Moser: Stable and random motions in dynamical systems. Ann. Math. Stud., vol. 77. Princeton, NJ: Princeton University Press 1973

[M2] Moser, J.: Is the solar system stable? Mathematical Intelligencer 1, 65-71 (1978)

[VS] Vivaldi, F., Shaidenko, A.: Global stability of a class of discontinuous dual billiards. Commun. Math. Phys. 110, 625-640 (1987) 
